ارزيابى عملكرد توليدمثلى ميشهاى افشارى با استفاده از روشى كوتاه

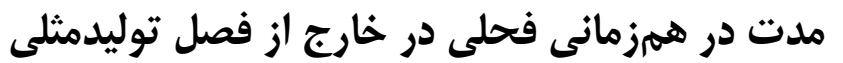

ير يسا حجازى'، رضا معصومى'، مجيد شاهمر ادى'، بهنام رستمى 'و مهيار باقرى نيا اميرى

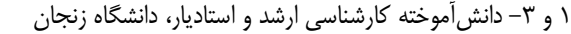

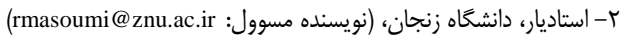

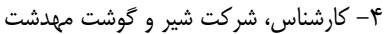

تاريخ يذيرش:

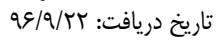

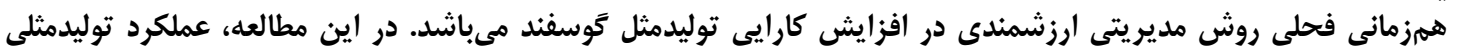

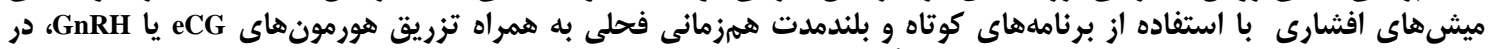

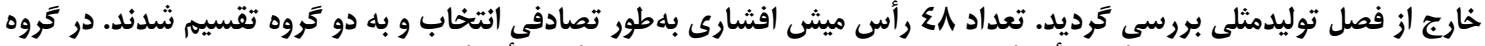

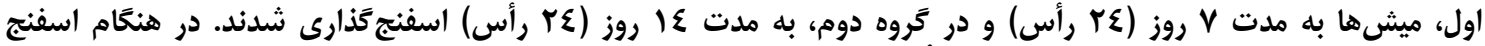

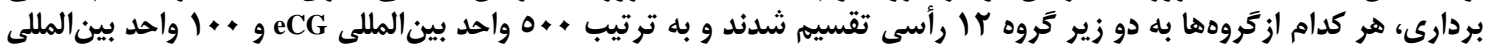

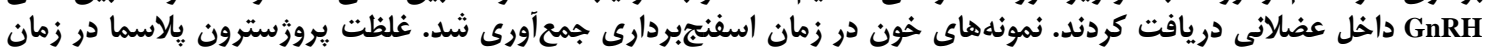

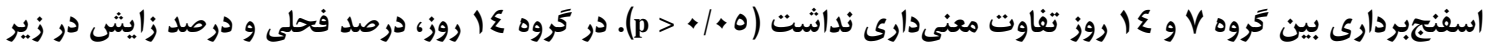

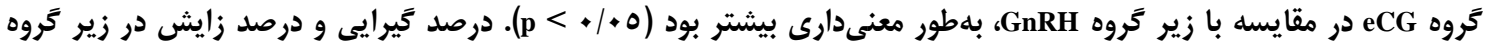

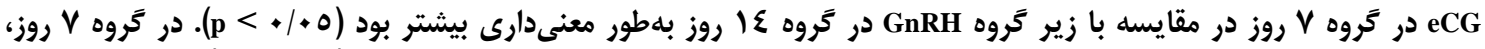

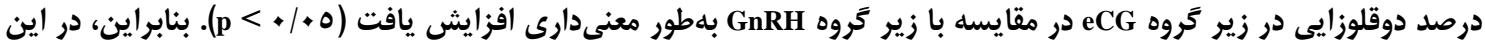

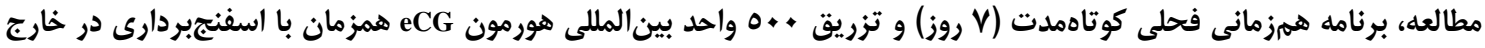

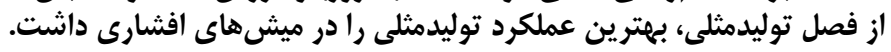

وازههاى كليدى: كَنادوترويين، همزمانى فحلى، ميش افشارى

همانند دورههاى بلندمدت آن در القا و همزمانسازى فحلى فرألى

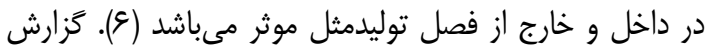

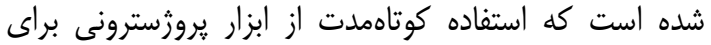

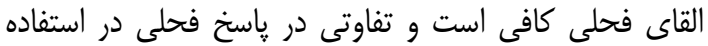

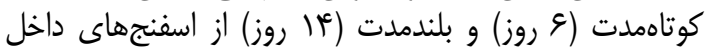

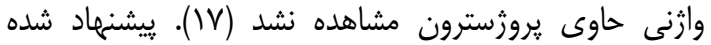

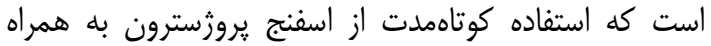

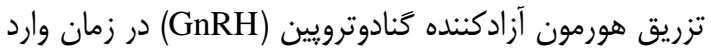

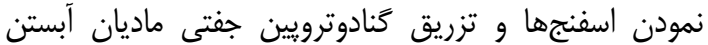
و و بروستاكًالندين (eCG)

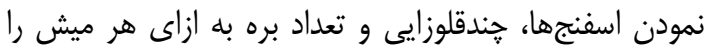

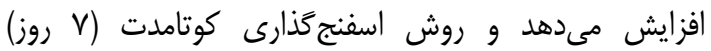

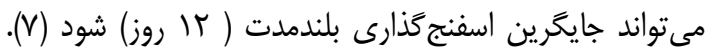

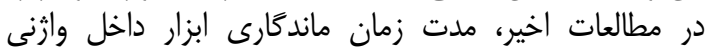

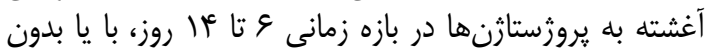

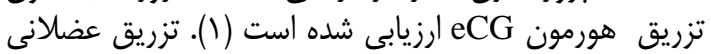

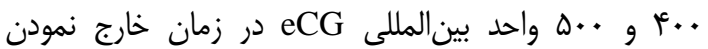

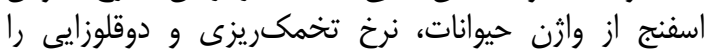

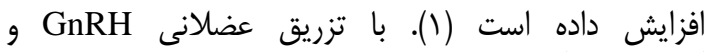

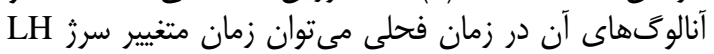

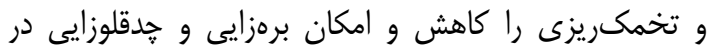

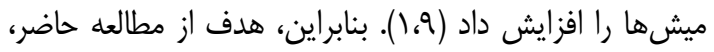

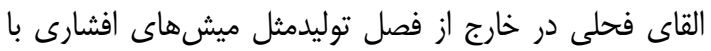

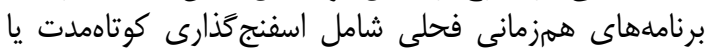

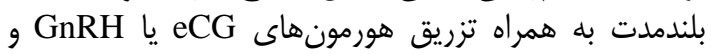

مقدمه

همزمانى فحلى ابزار مديريتى ارزشمندى است كه به به

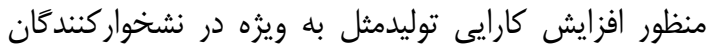

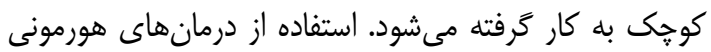

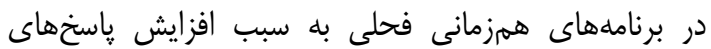

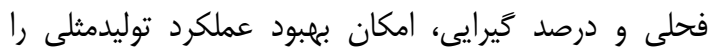

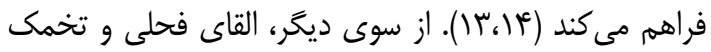

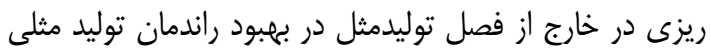

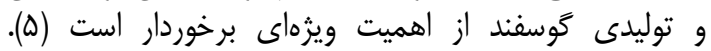

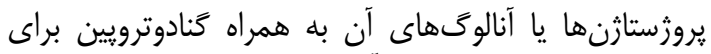

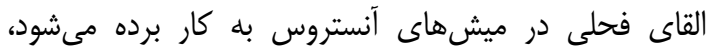

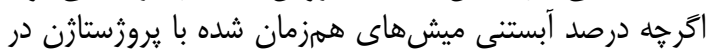

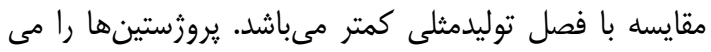

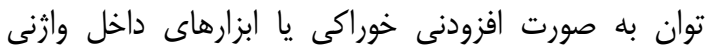

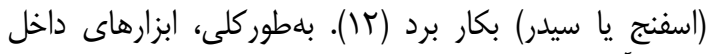

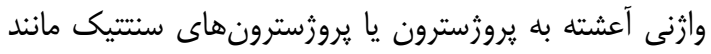

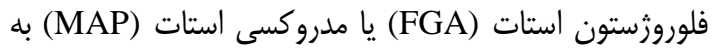

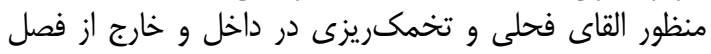

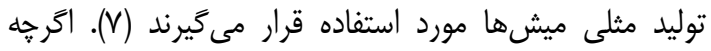

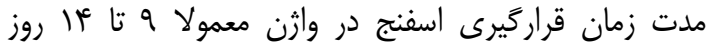

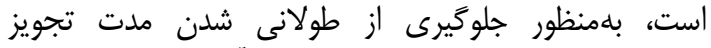

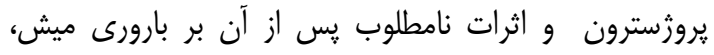

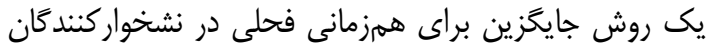

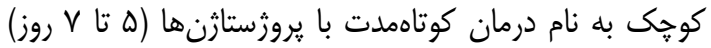

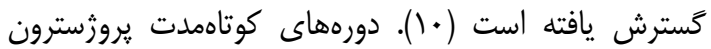


ميانكين توسط آزمون مربع كاى (chi-squire) آناليز شد و غلظت برورسترون با استفاده از رويه GLM تجزيه واريانس

شد.

$\mathrm{Y}_{\mathrm{ijk}}=\mu+\mathrm{A}_{\mathrm{i}}+\mathrm{B}_{\mathrm{j}}+\mathrm{C}_{\mathrm{k}}+\mathrm{e}_{\mathrm{ijkl}}$

年

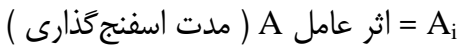

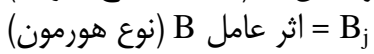

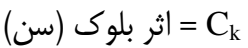

خطاى آزمايش

نتايج و بحث

نتايج مطالعه حاضر نشان داد كه دورهمهاى متفاوت بتاوت

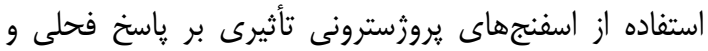

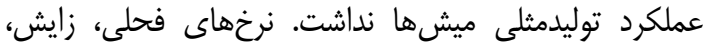

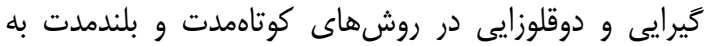

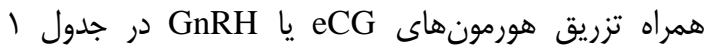

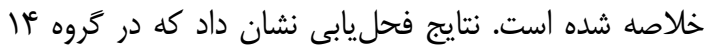

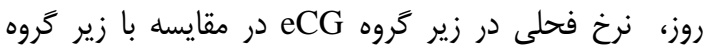

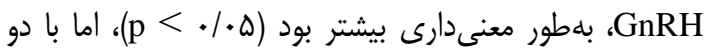

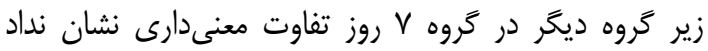

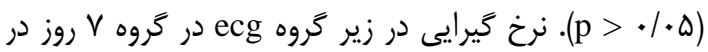

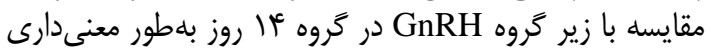

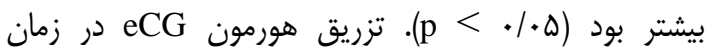

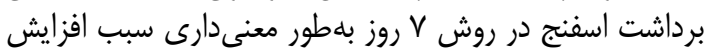
نرخ زايش در مقايسه با تزريق هورمون

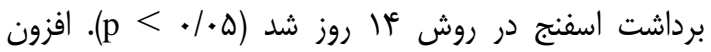

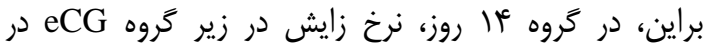
مقايسه با زير كروه

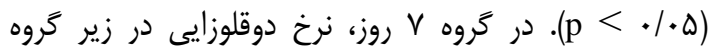

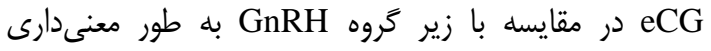

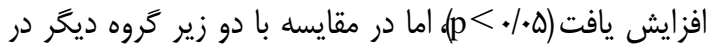

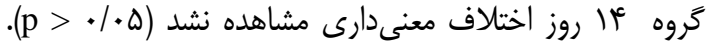

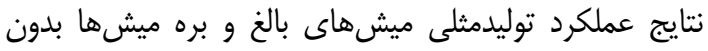

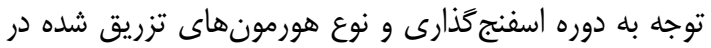

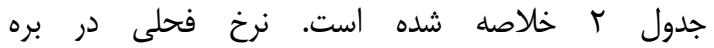

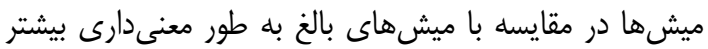

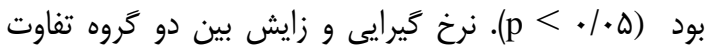

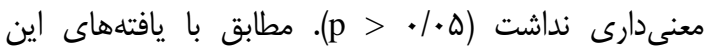

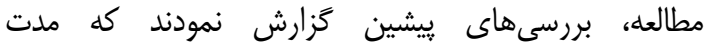

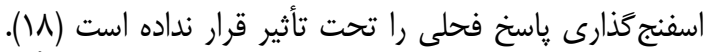

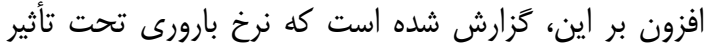

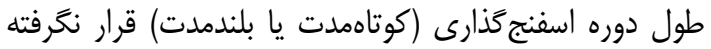

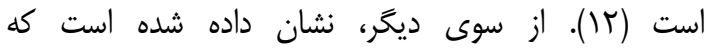

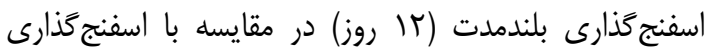

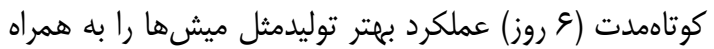

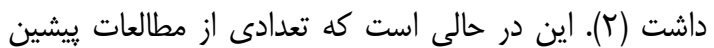

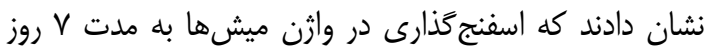

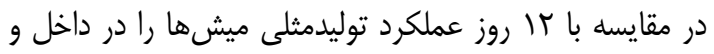

امكان جايخزينى هورمون GnRH با eCG در زمان اسفنج

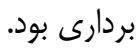

مواد و روشها

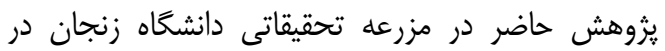

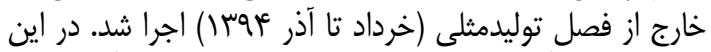

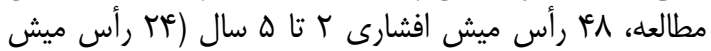

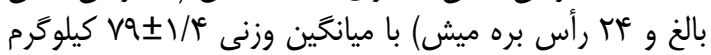

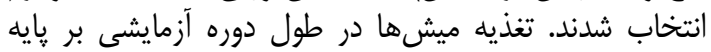

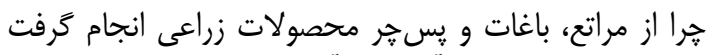

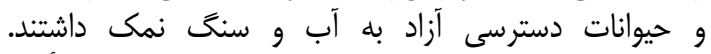

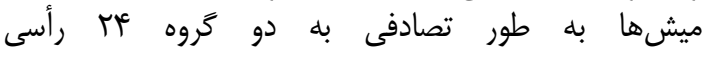

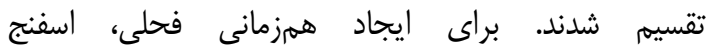

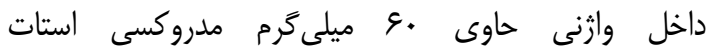
Medroxyprogesterone acetate/Sponge, intervet, )

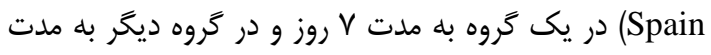

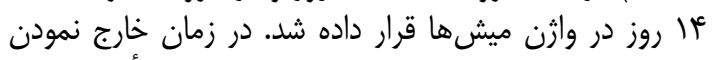

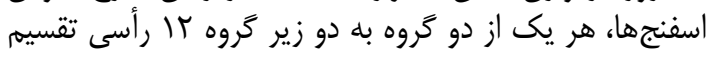

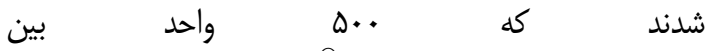
المللى (GONASER ${ }^{\circledR}$; Hipra; Spain) eCG به زير كروه

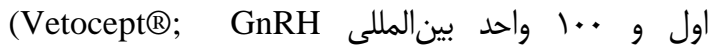
Aburaihan; Iran)

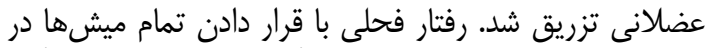

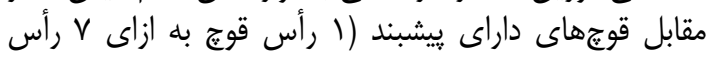

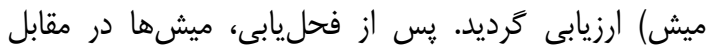

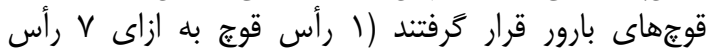

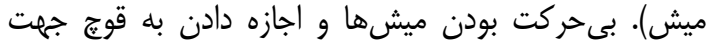

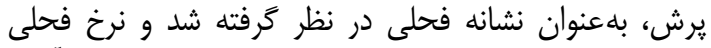

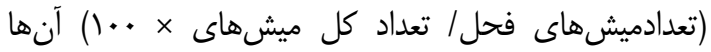

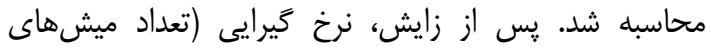

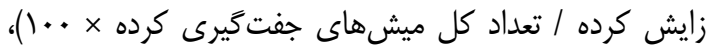

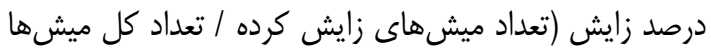

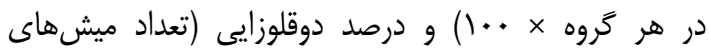

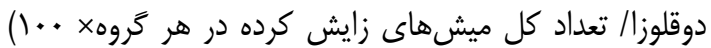

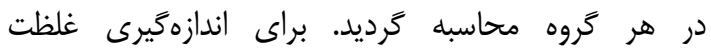

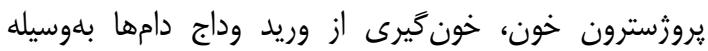

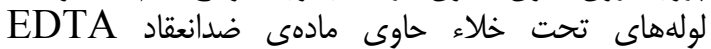
در زمان (Ethylene Diamine Tetra Acetic Acid)

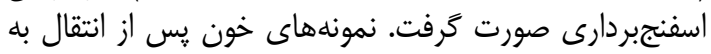

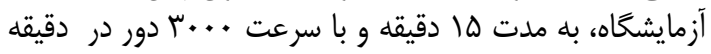

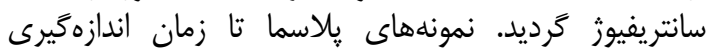

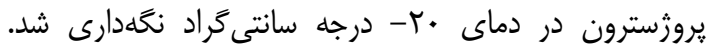

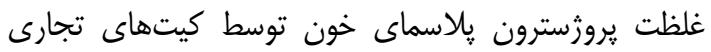

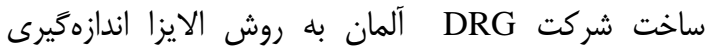

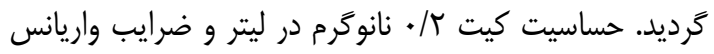

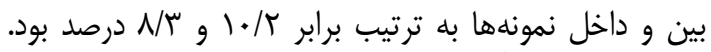

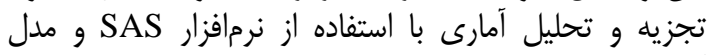

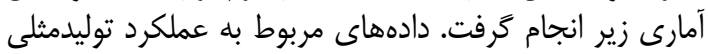
با استفاده از روش لوجستيك، رويه GENMOD و و مقادهايسات 
سوى ديخر، استفاده كوتاه مدت يرورستازن (9 روز) منجر به إنها

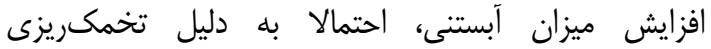
فوليكولهاى در حال رشد جديد مىباشد (19).
خارج از فصل توليدمثلى بهبود مى بخشد (بآ). نرخ بايين

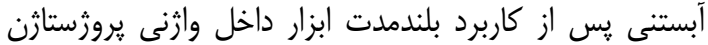

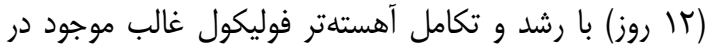

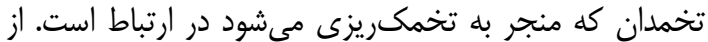

جدول ا- اثر مدت اسفنج گذارى و نوع كنادوترويين تزريق شده در زمان خارج نمودن اسفنج داخل واثنى بر عملكرد توليدمثل ميشهاى افشارى (ميانكين SEM

Table 1. The effect of sponge duration and type of gonadotropin at the time of sponge removal on reproductive performance of Afshari ewes

\begin{tabular}{|c|c|c|c|c|c|c|}
\hline \multirow[t]{2}{*}{ P-Value } & \multirow[t]{2}{*}{ SEM } & \multicolumn{2}{|c|}{ F } & \multicolumn{2}{|c|}{ V V روز V } & \\
\hline & & GnRH & eCG & GnRH & eCG & \\
\hline$\cdot 1 \cdot 1$ & $11 / \mathrm{V}$ & $\begin{array}{c}\Delta \cdot / \cdot \cdot \cdot \\
(\xi / N T)^{b}\end{array}$ & $\begin{array}{l}91 / 9 V \\
(11 / 1 \%)^{\mathrm{a}}\end{array}$ & $\begin{array}{l}V D / . . \\
(q / / T)^{\mathrm{ab}}\end{array}$ & $\begin{array}{c}\wedge r / r r \\
(1 \cdot / \mu)^{\mathrm{ab}}\end{array}$ & درصد فحلى \\
\hline .1 .0 & $\mid r / . \cdot$ & $\begin{array}{l}\Delta \cdot / \cdot \cdot \\
(\Gamma / \varepsilon)^{b}\end{array}$ & $\begin{array}{c}11 / 11 \\
(\mathrm{q} / 11)^{\mathrm{ab}}\end{array}$ & $\begin{array}{l}q q / q \mathrm{~V} \\
(q / 9)^{\mathrm{ab}}\end{array}$ & $\begin{array}{l}q \cdot / \cdot \cdot \\
(q / 1 \cdot)^{a}\end{array}$ & نرخ كَيرايى \\
\hline r./. & $\mid r / \cdot$ & $\begin{array}{l}r \Delta /: \bullet \\
(r / l r)^{b}\end{array}$ & $\begin{array}{l}V Q /: \cdot \\
(Q / / T)^{a}\end{array}$ & $\begin{array}{l}a \cdot / \cdot \cdot \\
(\xi / \mid r)^{\mathrm{ab}}\end{array}$ & $\begin{array}{l}V Q / . . \\
(Q / I T)^{a}\end{array}$ & درصد زايش \\
\hline.$/ . r$ & $\mid \mathrm{V} / \cdot \cdot$ & $\begin{array}{l}r / / r \\
(1 / r)^{\mathrm{ab}}\end{array}$ & $\begin{array}{l}r / / r r \\
(T / q)^{a b}\end{array}$ & $(\cdot / \varepsilon)^{b}$ & $\begin{array}{l}V V / V V \\
(V / q)^{a}\end{array}$ & درصد دوقلوزايى \\
\hline
\end{tabular}

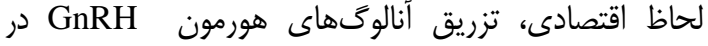

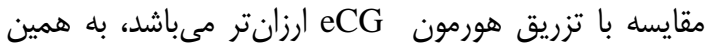

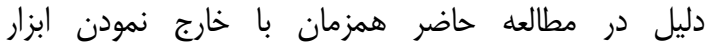

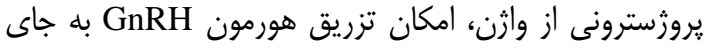

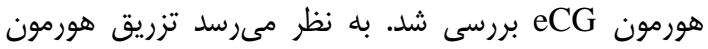
ت تخك eCG

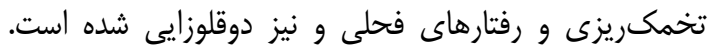

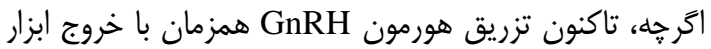

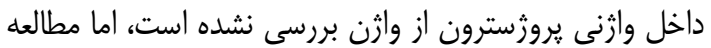

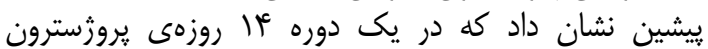

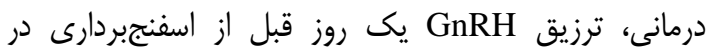

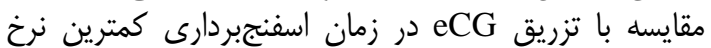

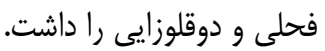

اخلاف در نتايج آزمايشها ممكن است به دليل تفاوت در

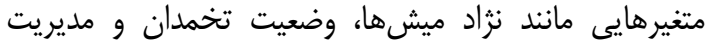

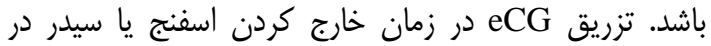

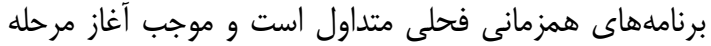

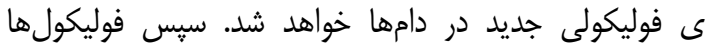

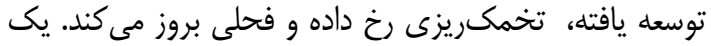

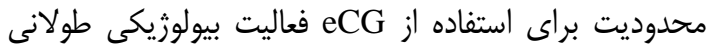

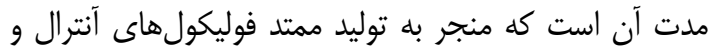
در نتيجه باعث توليد تعداد زيادى فوليكولهای آنهاى آترتيك

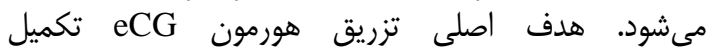

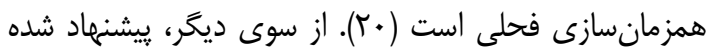

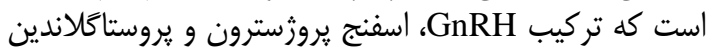

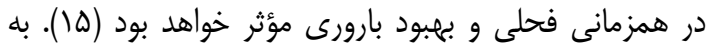

جدول r- مقايسه عملكرد توليدمثلى در ميشهاى بالغ و بره ميشها (ميانگين SEM Table 2. Reproductive performance of mature ewes and ewe lambs

\begin{tabular}{|c|c|c|c|c|}
\hline P-Value & S.E.M & بره ميش & ميش بالغ & \\
\hline & & $u^{e}$ & re & تعداد ميش در آزمايش \\
\hline & & rI & 10 & تعداد فحلى \\
\hline \multirow[t]{2}{*}{$.1 \cdot f$} &.$/ F F$ & $\Lambda V / \Delta \cdot(\Gamma) / T F)^{\mathrm{a}}$ & $G K / \Delta \cdot(1 Q / T Y)^{D}$ & فحلى (\%) \\
\hline & & is & 11 & تعداد زايش \\
\hline זr/. & .111 & $g \& / G V(\mid S / M Y)$ & $F \Delta / \Lambda \Gamma(I I / T F)$ & زايش (\%) \\
\hline 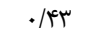 & $\cdot|\Delta|$ & $V \& / 19(\mid g / r))$ & VT/K $(11 / 1 Q)$ & نرخ گيرايى (\%) \\
\hline
\end{tabular}

حروف متفاوت هر رديف تفاوت معنىدار را نشان مىدهد ( ه./. > p).

كيرايى و زايش در بره ميشها مشاهده شد. برخلاف نتايج

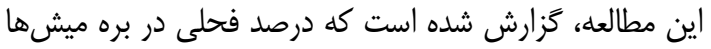

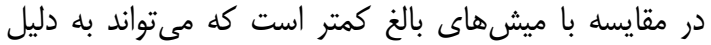

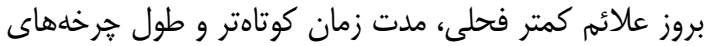

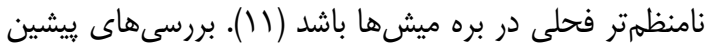

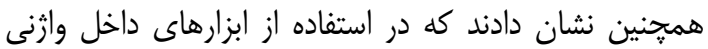

بهترين عملكرد توليدمثلى در اين مطالعه با روش

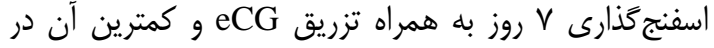

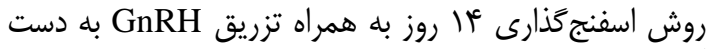

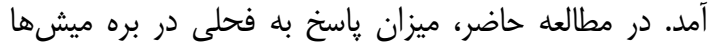

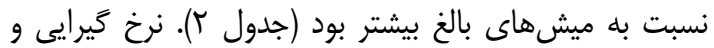
زايش بين دو گروه تفاوت معنىدار نداشت اما بيشترين نئ نرخ 
قلو با دوقلو در كروههاى آزمايشى مورد مقايسه قرار نغخرفت.

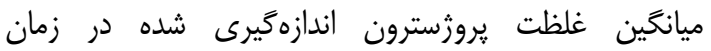

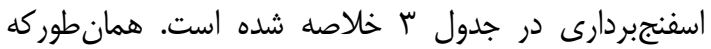

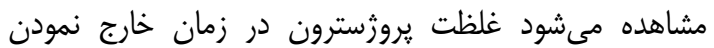

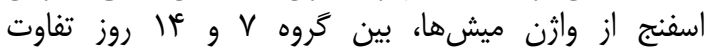

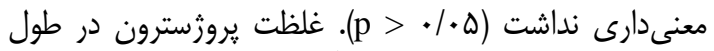

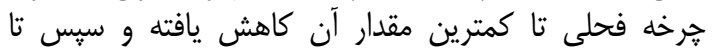

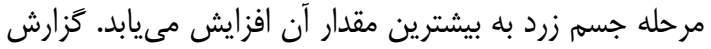

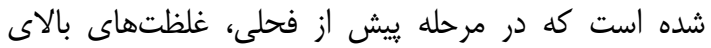

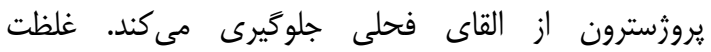

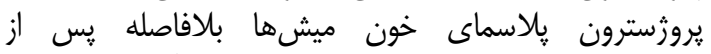

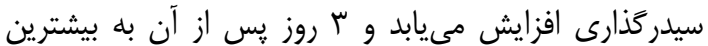

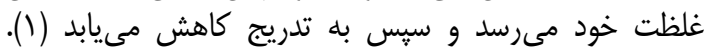

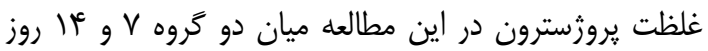
تفاوت معنى دارى نداشت.

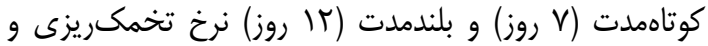

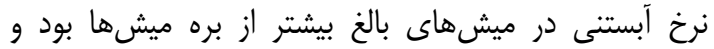

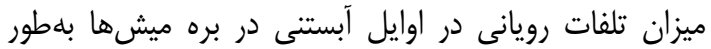

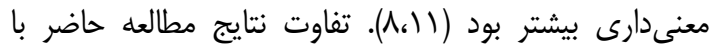

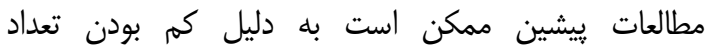

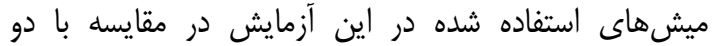

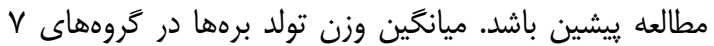
روز $\mathrm{GnRH}+j g$ رو $1 f$ و

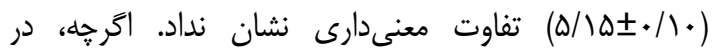

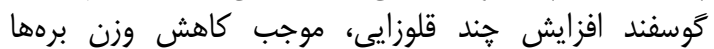

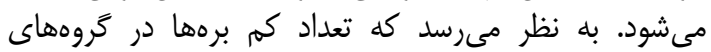

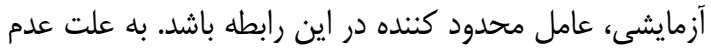
وجود دو قلوزايى در كروه V V روز

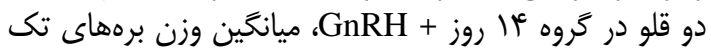

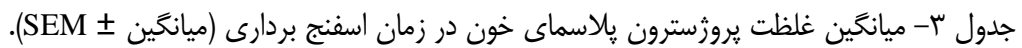
Table 3. The mean concentration of plasma progesterone at the time of sponge removal

\begin{tabular}{|c|c|c|c|c|}
\hline P-Value & SEM & F & V & \\
\hline & & $M F$ & $M r$ & تعداد ميش \\
\hline .1 .9 & .199 & ع/r & r/er & קروزسترون \\
\hline
\end{tabular}

گرديد. با توجه به كوتاه بودن طول دوره اين برنامه همزمانى

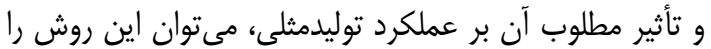

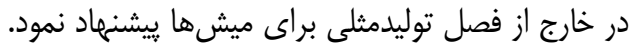

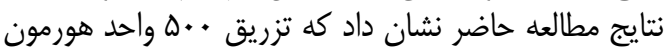
eCG

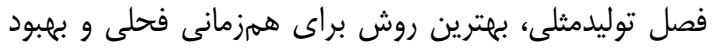

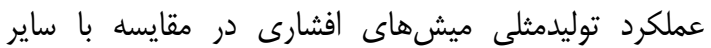
روشهاى استفاده شده در اين مطالعه بود. به اين وسيله از جناب آقاى دكتر داود كوليوند در صفحه

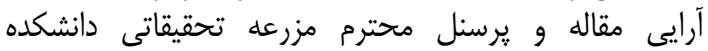

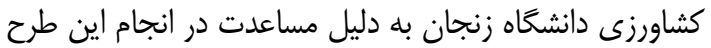
سياسگزارى مىشود.
اين روش، با توجه به كاهش مدت زمان استفاده از إن إنان

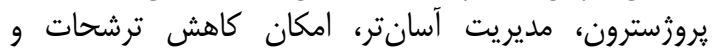

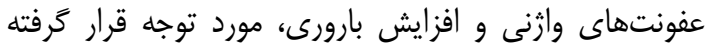

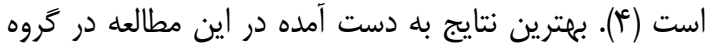

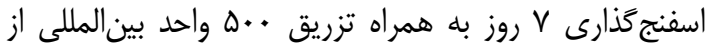

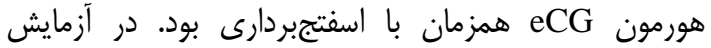

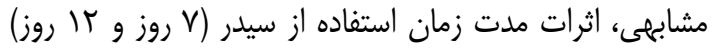

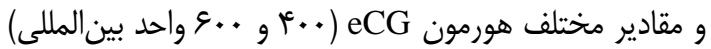

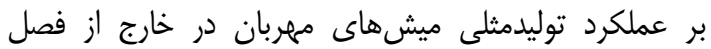

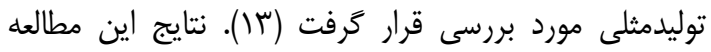

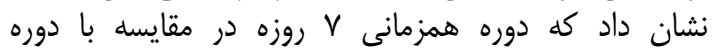
همزمانى

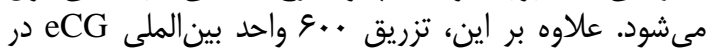

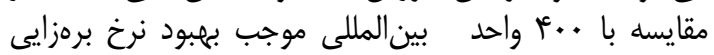


1. منابع Khalil and M.S. Abdel-Fattah. 2014. Effect of progestagen. PGF2 $\alpha$. PMSG AND GnRH on estrus svnchronization and some reproductive and productive traits in Barki ewes. Journal of Agroalimentarv Processes and Technologies. 20: 93-101.

2. Ahmed Amer. A. and A. Maher Hazzaa. 2009. The effect of different progesterone protocols on the reproductive effciencv of ewes during the non-breeding season. Veterinarski arhiv. 79: 19-30.

3. Ataman. M.B.. M. Akoz and O. Akman. 2006. Induction of svnchronized oestrus in Akkaraman crossbred ewes during breeding and anestrus seasons: the use of short-term and long-term progesterone treatments. Revue de médecine vétérinaire. 157-257.

4. Fonseca. J.F.. J.H. Bruschi. I.C. Santos. J.H. Viana and A.C. Magalhães. 2005. Induction of estrus in non-lactating dairy goats with different estrous synchrony protocols. Animal Reproduction Science, 85: 117-124.

5. Hashemi, M., M. Safdarian and M. Kafi. 2006. Estrous response to synchronization of estrus using different progesterone treatments outside the natural breeding season in ewes. Small Ruminant Research, 65: 279-283.

6. Husein, M.Q., M.M. Ababneh and D.S. Abu-Ruman. 2007. The effects of short or long term FGA treatment with or without eCG on reproductive performance of ewes bred out-of-season. American Journal of Animal and Veterinary Sciences, 2: 23-28.

7. Karaca, F., M.B. Ataman and K. Coyan. 2009. Synchronization of estrus with short-and long-term progestagen treatments and the use of GnRH prior to short-term progestagen treatment in ewes. Small Ruminant Research, 81: 185-188.

8. Martinez, M.F., B. McLeod, G. Tattersfield, B. Smaill, L.D. Quirke and J.L. Juengel. 2015. Successful induction of oestrus, ovulation and pregnancy inadult ewes and ewe lambs out of the breeding season using a GnRH + progesterone oestrus synchronization protocol. Animal Reproduction Science, 155: 28-35.

9. Mehri. R.. B. Rostami. R. Masoumi and M.H. Shahir. 2018. Effect of iniection of GnRH and hCG on dav 5 post mating on maternal P4 concentration and reproductive performance in Afshari ewes. Journal of Comparative Pathobiolog, 14: 2363-2370.

10. Metodiev, N. and E. Raicheva. 2011. Effect of the short-term progestagen treatments plus PMSG prior ram introduction on the estrus synchronization and the fertility of Ile de France ewes. Biotechnology in Animal Husbandry, 27: 1157-1166.

11. Mulvaney, F.J., S.T. Morris, P.R. Kenyon, P.C.H. Morel, D.M. West, C. Vi Noles and K.M.M. Glover. 2013. Comparison between the reproductive performance of ewe hoggets and mature ewes following a progesterone-based oestrus synchronization protocol. New Zealand Journal of Agricultural Research, 56: 288-296.

12. Ozyurtlu, N., S. Ay Serhan, I. Kucukaslan, O. Gungor and S. Aslan. 2011. Effect of subsequent two short-term, short-term, and long-term progestagen treatments on fertility of Awassi ewes out of the breeding season. Ankara Üniversitesi Veteriner Fakültesi Dergisi, 58: 105-109.

13. Sareminejad, P., S. Tabatabaei, M. Mamouei, K. Mirzadeh and M. Boujarpour. 2014. The Effects of short and long term medroxy Progesterone acetate (MAP) sponge treatments on reproductive performance during the non-breeding season of Arabian ewes. Iran. Journal of Applied Animal Science. 4: 747-751.

14. Shahneh. A.Z.. H.D. Taiangookeh. H.S. Panah and A.A. Saki. 2006. Effect of controlled internal drug release device treatment duration and eCG dose on reproductive performance of seasonally anestrous fat-tailed Iranian ewes. Pakistan Journal of Biological Sciences, 9: 1552-1555.

15. Titi, H.H., R.T. Kridli and M.A. Alnimer. 2010. Estrus synchronization in sheep and goats using combinations of GnRH, progestagen and prostaglandin F2 $\alpha$. Reproduction in Domestic Animals, 45: 594-599.

16. Towhidi. A.. R. Masoumi. M.M. Moeini. H. Solgi and H. Moravei. 2007. The relationship between plasma leptin and FSH concentrations with ovulation rate in Iranian native sheep. Pakistan Journal of Biological Sciences, 10: 363-367.

17. Ungerfeld, R. and E. Rubianes. 2002. Short term primings with different progestogen intravaginal devices (MAP, FGA and CIDR) for eCG oestrous induction in anestrus ewes. Small Ruminant Research, 46: 63-66.

18. Ustuner, B., U. Gunay, Z. Nur and H. Ustuner. 2007. Effects of long and short-term progestagen treatments combined with PMSG on oestrus synchronization and fertility in Awassi ewes during the breeding season. Acta Veterinaria Brno, 76: 391-397.

19. Vinoles, C., M. Forsberg, G. Banchero and E. Rubianes. 2001. Effect of long-term and short-term progestagen treatment on follicular development and pregnancy rate in cyclic ewes. Theriogenology, 55: 993-1004.

20. Wheaton, J.E., K.M. Carlson, H.F. Windels and L.J. Johnston. 1993. CIDR, a new progesterone releasing intravaginal device for induction of estrous and cycle control in sheep and goats. Animal Reproduction Science, 33: 127-141. 


\title{
Evaluation of Reproductive Performance of Afshari Ewes with a Short Estrus Synchronization Program in Non Breeding Season
}

\author{
Parisa Hejazi $^{1}$, Reza Masoumi ${ }^{2}$ Majid Shahmoradi ${ }^{3}$, Behnam Rostami ${ }^{3}$ \\ and Mahyar Bagherinia Amiri ${ }^{5}$ \\ 1 and 3- Graduated M.Sc. Student and Assistant Professor, University of Zanjan \\ 2- Assistant Professor, University of Zanjan (Corresponding Author: rmasoumi@ znu.ac.ir) \\ 4- Mahdasht Dairy Corporation \\ Received: December 13, 2017 \\ Accepted: May 1, 2018
}

\begin{abstract}
Estrus synchronization is a valuable management tool that has been employed in enhancing reproductive efficiency in ewes. This study was conducted to investigate the reproductive performance of ewes with short and long-term estrous synchronization programs accompanied by eCG or GnRH during the non-breeding season. A total of 48 Afshari ewes were randomly selected and divided into two groups. In the first group, ewes received intravaginal sponges for 7 days $(n=24)$ and in the second group ewes received intravaginal sponges for 14 days $(n=24)$. At the time of sponge removal, each group was divided into two subgroups of 12 ewes; intramuscularly received $500 \mathrm{IU}$ eCG and $100 \mathrm{IU} \mathrm{GnRH}$, respectively. Blood samples were collected at the time of sponge removal. Considering plasma progesterone concentrations, there were no significant differences between 7 and 14 days groups $(\mathrm{P}>0.05)$. In 14 days group, estrus and lambing rates of eCG subgroup were significantly higher than those in GnRH subgroup (P $<0.05)$. Conception and lambing rates of eCG subgroup in 7 days group were significantly higher than those in GnRH subgroup in 14 days group $(\mathrm{P}<0.05)$. In 7 days group, twinning rate of eCG subgroup was significantly higher than that of GnRH subgroup $(\mathrm{P}<0.05)$. Therefore, in this study, short-term estrus synchronization programs (7 days) and injection of $500 \mathrm{IU}$ eCG simultaneous with sponge removal exhibited the best reproductive performance in Afshari ewes during non-breeding season.
\end{abstract}

Keywords: Afshari ewe, ECG, GnRH, Non- breeding, Short estrus synchronization 